\title{
Organic photovoltaic cells: Novel organic semiconducting materials and molecular arrangement engineering
}

\author{
WANG ZiXuan, ZHANG FuJun*, WANG Jin, XU XiaoWei, WANG Jian, LIU Yang \& XU Zheng
}

Key Laboratory of Luminescence and Optical Information (Ministry of Education), School of Science, Beijing Jiaotong University, Beijing 100044, China

Received November 2, 2011; accepted February 16, 2012; published online May 15, 2012

\begin{abstract}
Organic photovoltaic cells (OPVs) have attracted more and more attention due to its highly potential application to solve the energy crisis considering its advantages, such as low cost and ease of large area production. The power conversion efficiency (PCE) of OPVs has undergone a more than nine-fold increase from $1.0 \%$ by Tang in 1986 to $9.2 \%$ in 2010 announced by Mitsubishi Chemical. The major challenges of obtaining high efficiency OPVs are the synthesis of new narrow band gap materials, controlling molecular arrangement, designing novel configuration cells for better photon harvesting in the active layer. In the article, we summarized the recent progress of novel narrow band gap photovoltaic materials and the effective methods to control the morphology of donor and acceptor in the blend films for high performance of OPVs.
\end{abstract}

photovoltaic, narrow band gap materials, molecular arrangement

Citation: Wang Z X, Zhang F J, Wang J, et al. Organic photovoltaic cells: Novel organic semiconducting materials and molecular arrangement engineering. Chin Sci Bull, 2012, 57: 4143-4152, doi: 10.1007/s11434-012-5202-3

Solar light is the most important renewable and environmentally friendly energy, which can be converted into electrical energy in a photovoltaic cell. Silicon solar cells have high power conversion efficiency (PCE), long durability and the benefit of several decades of research and development. However, silicon metallurgy has a great impact on the environment and the input-output ratio of silicon solar cells is still low. These problems have prompted the seeking for alternative photovoltaic cells based on other inorganic semiconductors or organic materials. Recently, organic photovoltaic cells (OPVs) have attracted more and more attention as new energy source due to their light weight, ease of large scale manufacture, compatibility with flexible substrates, and the need of developing an inexpensive clean and sustainable renewable energy source to satisfy economic development and human living [1-5]. Great efforts have been devoted to the design and synthesis of new conjugated polymer donor and new fullerene derivative acceptor mate-

*Corresponding author (email: fjzhang@bjtu.edu.cn) rials for the better matching with the solar light spectra. The main contribution of the photon harvesting in the OPVs should be attributed to the conjugated polymer donor, and the fullerene derivates just play a slight role in photon harvesting. From the side of organic semiconducting materials, the suitable band gap and high charge carrier mobility will determine the open circuit voltage $\left(V_{\text {oc }}\right)$ and short circuit current $\left(J_{\mathrm{sc}}\right)$ of OPVs. The most straightforward way to reduce the band gap is simply by either raising the highest occupied molecular orbits (HOMO) or reducing the lowest unoccupied molecular orbits (LUMO) level of the polymer or by compressing the two levels closer simultaneously. The energy level of accepter material should be taken into account for the higher PCE. It is known that $V_{\text {oc }}$ is determined by the energy levels difference between the HOMO of the electron donor and the LUMO of electron acceptor. So the lower HOMO energy level of an electron donor will benefit $V_{\text {oc }}$, whereas reduction in a polymer's band gap by lifting up the HOMO level will inevitably result in a loss of $V_{\text {oc }}$. On the other hand, the LUMO energy level of donor materials 
has to be at least $0.3 \mathrm{eV}$ higher than that level of the fullerene derivatives to guarantee the formation of a downhill driving force for the charge carriers' separation and transfer [6]. So reduction in a polymer's band gap by pushing down the LUMO level will inevitably result in a loss of $J_{\mathrm{sc}}$, which is related to the charge carrier transfer and the absorption in the visible light range. The larger band gap of the donor will produce a relatively large $V_{\text {oc }}$ and relatively small $J_{\text {sc }}$, the lower band gap of electron donor will result in a relatively large $J_{\mathrm{sc}}$ and relatively small $V_{\mathrm{oc}}$. However, the product of $J_{\mathrm{sc}}$ and $V_{\mathrm{oc}}$ will determine the final performance of cells, making the balanced $J_{\mathrm{sc}}$ and $V_{\mathrm{oc}}$ showing a better performance. It is known that most polymer materials have a linear chemical structure with a large ratio of length to width. So the molecular arrangement will strongly influence its optical absorption and charge carrier transport. From the molecular engineering view, a series of research works on controlling the morphology of donors and acceptors have been carried out to optimize the exciton diffusion, dissociation and charge carrier transport in the bulk heterojunction OPVs, such as annealing treatment, electric field treatment, inserting interfacial buffer layer, glance angle deposition methods.

The conventional OPV cells' architecture was made of a single active bulk heterojunction layer or planner junction layers sandwiched by a high work function and transparent metal oxide as the bottom anode and a low work function metal as the top cathode. In order to widen the solar spectra response range, tandem configuration of OPVs was fabricated with two or more active layers made from different band gap materials for a better match with solar light $[7,8]$. Recently, a kind of inverted configuration OPVs with low work function metals modified ITO as the transparent cathode and high work function metals as anode was proposed to improve the stability of the cell and avoid the cell's performance degradation induced by oxidation of metal cathodes under room conditions $[9,10]$. In this article, we summarized the recent development of OPVs from two sides, novel narrow band gap materials and molecular engineering.

\section{Organic photovoltaic materials}

\subsection{Electron donating semiconducting materials}

The main losses of photo-electric conversion are the photon loss, exciton loss and charge carrier loss in the OPVs. These losses strongly depend on the materials' characteristics, including band gap, exciton diffusion length, and charge carrier mobility. Up to now, the performance of photovoltaic cells with these conjugated polymers is considerably limited by their relatively large band gaps, which result in the mismatch between the absorption spectrum of the active layer and the solar light spectrum, especially in the red and nearinfrared ranges. In order to further improve the PCE of the OPVs, many research works have been devoted to synthesis new materials or modify former ones aiming at a broader absorption, lower band gap, higher hole mobility, and more suitable electronic energy levels matched with the acceptor energy level for efficient excition separation. Therefore, the development of low band gap donor polymers is of crucial importance for increasing PCE of OPVs. Poly(3-hexylthiophene) (P3HT) has been extensively studied and results in a great improvement over the first widely studied material MEH-PPV. It has a good solubility, environmental stability, a relatively lower band-gap and higher absorption edge around $650 \mathrm{~nm}$ combined with a high hole mobility exceeding $0.1 \mathrm{~cm}^{2} / \mathrm{Vs}$ [11]. Hiorns et al. [12] synthesized a series of poly(3-hexylthiophene)s (P3HTs) and poly(3-butylthiophene)s (P3BTs) with predetermined molecular weights and varying polydispersities. It is interesting that their electrochemical band gaps were slightly decreased along with the increase of molecular weight. The results indicate that the polydispersities, molecular weights, and degrees of conjugation have an important impact on not only cell characteristics but also the most effective annealing temperature required.

Various band gap materials have been synthesized in order to better match with the solar light spectrum [13-26], which are summarized in Figure 1. The detailed parameters of materials and OPVs' performance are listed in Table 1. Among the novel electron donating materials, PCDTBT presents a great potential in the actual application due to its narrower band gap $(1.83 \mathrm{eV})$ with the HOMO level at $-5.15 \mathrm{eV}$ and LUMO level at $-3.32 \mathrm{eV}$, respectively. The lower HOMO of PCDTBT compared with P3HT (-4.7 eV) leads to the increased $V_{\text {oc }}$ and better chemical stability. The PCE of OPVs based on PCDTBT: PCBM as the active layer is about $7 \%$, with internal quantum efficiencies approaching $100 \%$ [14-16]. For the better photon harvesting in the active layer, $\mathrm{PC}_{71} \mathrm{BM}$ was selected to be the electron withdrawing material due to its relatively strong absorption in the visible light range. The $7.1 \%$ for $\mathrm{PCE}$ of $\mathrm{PCDTBT} / \mathrm{PC}_{71} \mathrm{BM}$ as the active layer was achieved [18]. Due to the limited exciton diffusion in organic semiconductors, the typical P3HT:PCBM system needs to be annealed to form an interpenetrating network for the efficient excition separation at the donor/acceptor interface. However, Beiley et al. [19] found that OPVs based on PCDTBT as donor material have a relatively high performance without annealing treatment. This finding indicates that much more attention should be paid to clarify the relationship between performance of OPVs and the structural order in the active layer.

He and Liang [21,27] developed a series of new semiconducting polymers based on alternating ester substitutes thieno[3,4-b]thiophene and benzodithiophene units. These polymers exhibit a synergistic combination of properties that lead to an excellent photovoltaic effect. Among them, PTB7 shows an excellent photovoltaic effect due to its strong absorption in the range from 550 to $700 \mathrm{~nm}$ corresponding to its HOMO $(-5.15 \mathrm{eV})$ and LUMO $(-3.31 \mathrm{eV})$ energy levels [21]. In this system, the exciton dissociation, the charge 


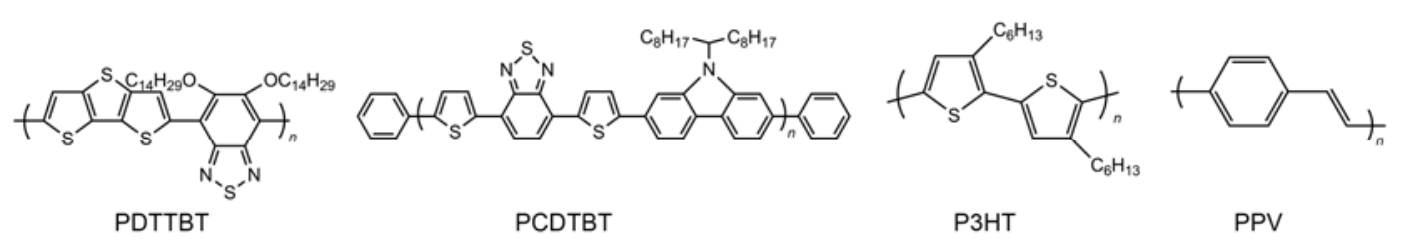

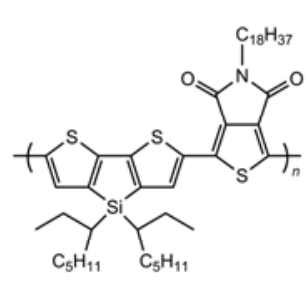

PDTSTBD

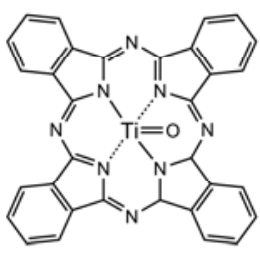

TiOPc

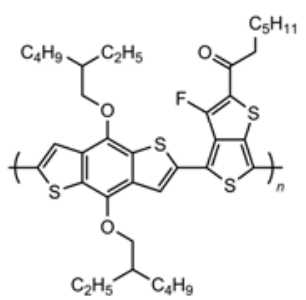

PBDTTT-CF

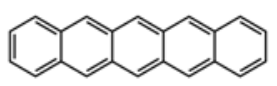

Pentacene

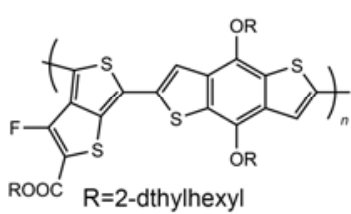

PTB7

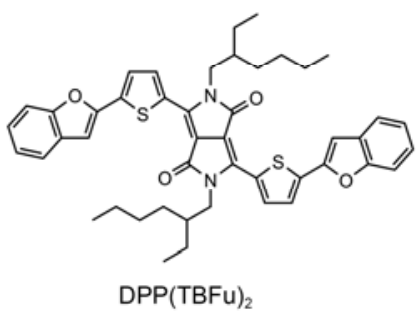

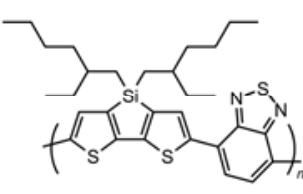

PSBTBT

Figure 1 Chemical structures of typical electron donating materials [13-26].

Table 1 The key parameters of band gap donor materials and their photovoltaic properties

\begin{tabular}{|c|c|c|c|c|c|c|c|}
\hline No. & Materials [donor:acceptor] & HOMO/LUMO $(\mathrm{eV})$ & $V_{\text {oc }}(\mathrm{V})$ & $J_{\mathrm{sc}}\left(\mathrm{mA} / \mathrm{cm}^{2}\right)$ & F.F. $(\%)$ & PCE $(\%)$ & Ref. \\
\hline 1 & P3HT:ICBA & $-4.7 /-2.8$ & 0.84 & 10.61 & 72.7 & 6.48 & [13] \\
\hline 2 & MEH-PPV:PCBM & $-5.1 /-2.7$ & 0.82 & 6.77 & 44.9 & 2.49 & [17] \\
\hline 3 & PCDTBT:PC ${ }_{71} \mathrm{BM}$ & $-5.15 /-3.32$ & 0.86 & 11.61 & 69.0 & 7.1 & [18] \\
\hline 4 & PTB7:PC ${ }_{71} \mathrm{BM}$ & $-5.15 /-3.31$ & 0.74 & 14.50 & 68.97 & 7.04 & [20] \\
\hline 5 & PTB7:PC ${ }_{71} \mathrm{BM}$ & $-5.15 /-3.31$ & 0.76 & 15.75 & 70.15 & 8.37 & [21] \\
\hline 6 & PDTSTPD:PC ${ }_{71} \mathrm{BM}$ & $-5.57 /-3.84$ & 0.89 & 14 & - & 7.3 & [22] \\
\hline 7 & PBDTTT-CF:PC ${ }_{71} \mathrm{BM}$ & $-5.22 /-3.45$ & 0.76 & 15.2 & 66.9 & 7.4 & [23] \\
\hline 8 & DPP:PC ${ }_{71} \mathrm{BM}$ & $-5.2 /-3.4$ & 0.92 & 10.0 & 48 & 4.4 & [24] \\
\hline 9 & TiOPC: $\mathrm{C}_{60}$ & $-5.7 /-3.9$ & 0.57 & 15.1 & 53 & 4.2 & [25] \\
\hline 10 & TPTPA: $\mathrm{C}_{70}$ & $-5.57 /-2.75$ & 0.90 & 3.6 & 66 & 2.2 & [26] \\
\hline
\end{tabular}

transport in donor and acceptor networks, and the charge extraction in both organic/electrode interfaces are very close to $100 \%$. These facts imply that the morphology of the blended films is close to the ideal donor and acceptor nanoscale interpenetrating network. He et al. [21] reported that simultaneous enhancement in the $J_{\mathrm{sc}}, V_{\mathrm{oc}}$, and FF was also demonstrated in PTB7:PC ${ }_{71} \mathrm{BM}$ OPVs when the poly [(9,9-bis(3'-( $N, N$-dimethylamino)propyl)-2,7-fluorene)-alt-2, 7-(9,9-dioctylfluorene)] (PFN) interlayer was incorporated as the electron selective layer. A PCE up to $8.37 \%$ for PTB7 as the electron donating material was obtained.

\subsection{Electron withdrawing semiconducting materials}

The most commonly used electron withdrawing materials are fullerene and fullerene derivatives, including $\mathrm{C}_{60}, \mathrm{C}_{70}$, $[6,6]$-phenyl $\mathrm{C}_{61}$-butyric acid methyl ester $\left(\mathrm{PC}_{60} \mathrm{BM}\right)$ and [6,6]-phenyl $\mathrm{C}_{71}$-butyric acid methyl ester $\left(\mathrm{PC}_{71} \mathrm{BM}\right)$ [28]. In 1992, Sariciftci et al. [29] firstly reported that $\mathrm{C}_{60}$ could efficiently quench MEH-PPV emissions by photoinduced electron transfer from the excited state of a conducting polymer onto buckminsterfullerene, $\mathrm{C}_{60}$, and related phenomena are not limited to MEH-PPV. After that, different structures of fullerene and its derivatives with various energy level alignment were doped into the polymer for high efficiently photoinduced electron transfer from the excited state of organic semiconducting materials [30-37]. These fullerene derivatives have many advantages in photovoltaic cells, including a deep-lying LUMO $(\sim 3.8-4.2 \mathrm{eV})$ for effective charge separation between the electron donor and acceptor, 
reversible reduction with ability to accept up to six electrons, ultra-fast three dimensional charge transfer, and high electron mobility.

As the heart of the OPV technology advantage is the ease of fabrication, which holds the promise of very low-cost manufacturing process. A simple choice is the solutionprocessed bulk heterojunction (BHJ) solar cell composed of electron-donating semiconducting polymers and electronwithdrawing fullerenes as active layers. The poor solubility of $\mathrm{C}_{60}$ limits its application in polymer solar cells. To solve this problem, Schinazi et al. [31] synthesized a soluble derivative of $\mathrm{C}_{60}$, PCBM (1-(3-methoxycarbonyl)propyl-1-phenyl $[6,6] \mathrm{C}_{61}$-butyric acid methyl ester) and applied it in OPV devices in 1993. This soluble fullerene derivative enabled the practical use of full solution process methods in preparing OPVs.

A series of fullerene derivatives with different diameter cage from $\mathrm{C}_{70}$ to $\mathrm{C}_{84}$ were used as electron acceptors in bulk heterojunction polymer-fullerene solar cells [32-37]. These fullerene derivatives have many advantages in photovoltaic cells, including a deep-lying lowest unoccupied molecular orbital (LUMO, $\sim 3.8-4.2 \mathrm{eV}$ ) for the effective charge separation between the electron donor and acceptor, reversible reduction with ability to accept up to six electrons, ultra-fast three dimensional charge transfer, and high electron mobility. Among them, $\mathrm{PC}_{70} \mathrm{BM}$ has strong absorption in the visible range, may compensate the absorption of many donor materials and significantly improve the performance of the devices [20]. Recently, He et al. [34] synthesized a series of new fullerene derivatives to enhance their absorption intensity in the visible light range, obtaining a significant improvement based on different kind of donor materials. What's more, some in-depth modification has been applied on the $\mathrm{C}_{60}$ itself. Chen et al. [35] synthesized a series of open-cage fullerenes possessing suitable solubilizing functionality with a variety of frontier molecular orbital energy levels and carrier mobility as n-type materials. Their experimental results pointed out a new direction of effective electron acceptor materials. However, fullerene and its derivatives have poor photochemical stability in the air and their spherical geometry leads to morphological instability in blend films with linear polymers, especially the vertical phase separation. Although fullerene materials are still the main research direction, non-fullerene materials have shown some very special features and great potential till now. Ren et al. [38] synthesized non-fullerene acceptor to obtain high efficiency of OPVs by engineering the nanomorphology via processing additives. The chemical structures of typical electron withdrawing materials are summarized in Figure 2.

\section{Molecular arrangement engineering}

One of the main challenges faced by the OPV community is

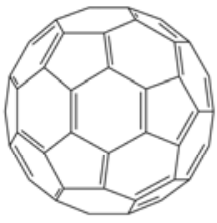

$\mathrm{C}_{60}$

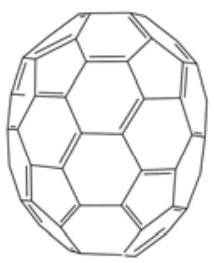

$\mathrm{C}_{70}$



$\mathrm{PC}_{60} \mathrm{BM}$

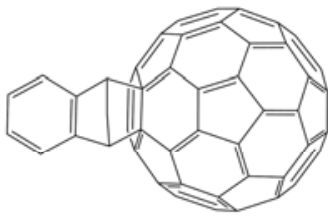

ICMA

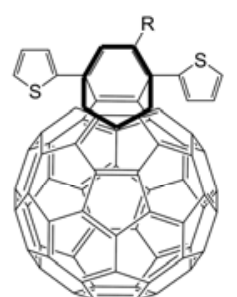

8-membered-ring fullerene

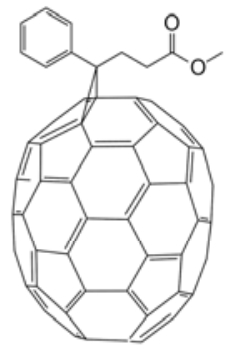

$\mathrm{PC}_{70} \mathrm{BM}$



ICBA

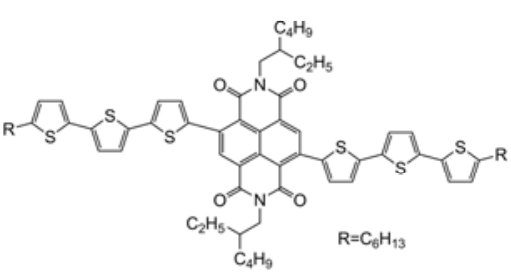

$\mathrm{NDI}-3 \mathrm{DH}$

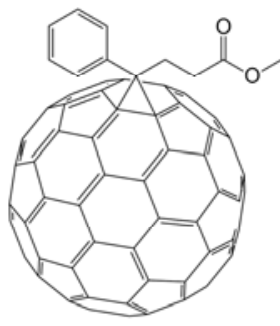

$\mathrm{PC}_{84} \mathrm{BM}$

Figure 2 Chemical structures of typical electron withdrawing materials [28,30,32,35,37,38]. 
the synthesis of narrow band gap semiconducting materials which can harvest more photon from sunlight. On that basis, forming donor/acceptor bicontinuous interpenetrating network is the most important factor to obtain high performance OPVs by controlling inter-molecular arrangement or morphology. Optimal blend morphology should consist a giving rise to a large amount of D/A interfaces due to the limited exciton diffusion length of $10 \mathrm{~nm}$, which is beneficial to the dissociation of photo-generated exciton. Meanwhile, the bicontinuous interpenetrating network could supply effective charge carrier transporting path to each individual electrode. Hence a delicate balance between phase separation and the continuous charge transport way is the key issue to obtain high efficiency OPVs. Material molecular arrangements and morphology strongly determine the performance of OPVs due to the characteristics dependence on the molecular arrangement, such as photon harvesting, morphology, charge carrier transport and collection. Various molecular arrangement engineering and interfacial engineering, such as annealing treatment, doping, interfacial buffer layer, different solvent treatment and electric field treatment methods, were carried out to control the molecular arrangement and morphology [39-44].

\subsection{Morphology of blend film adjusted by post treatment}

The active layers of polymer solar cells were needed to be treated to form donor/acceptor bicontinous interpenetrating network under different conditions, such as annealing treatment, electrical treatment as well as the solvent treatment. The annealing treatment is a very simple method, which places the substrate on a hot plate to accelerate dry of blend films. Both electron donor and acceptor molecules have an additional force to rearrange the film during the annealing treatment process. The molecular alignment could be adjusted during the volatilization of organic solvent from the active layer [45-47]. The effects of annealing treatment on the active layer or cell have been extensively carried out in order to improve the performance of PSCs, especially for the P3HT:PCBM system. The main contribution of annealing treatment could be concluded as the following points: (i) increase P3HT crystallization, which may affect its HOMO level and particularly the nature of thin film morphology near the film-air interface [47]; (ii) enhance P3HT absorption intensity and range and improve interchain and intrachain orders of P3HT [48]; (iii) reorganize and form a phase segregated 3D structure of donor and acceptor molecules enhancing the charge carrier transfer efficiency [49]; and (iv) optimize interfacial contact between the metal electrode and the active layer resulting in the increase of FF. [41]. In 2005, Ma et al. [50] firstly reported that the PCE of OPVs based on P3HT:PCBM was enhanced to $5 \%$ by post annealing treatment at $150^{\circ} \mathrm{C}$, which resulted from the pronounced improvement of $J_{\mathrm{sc}}$ and FF. Recently, we reported the effect of annealing treatment on the morphology and PL intensity of P3HT: $\mathrm{PC}_{60} \mathrm{Bm}$ and P3HT:PC ${ }_{70} \mathrm{BM}$ by optical microscopy under room conditions and green light excitation conditions [28]. The images are shown in Figure 3. There are some relatively large holes on the $\mathrm{P} 3 \mathrm{HT}: \mathrm{PC}_{60} \mathrm{BM}$ film surfaces compared with $\mathrm{P} 3 \mathrm{HT}: \mathrm{PC}_{70} \mathrm{BM}$ film surfaces, resulting in the larger surface roughness of P3HT:PC ${ }_{60} \mathrm{BM}$ film.

$\mathrm{Lu}$ et al. [51] also reported that different annealing treatment time on the performance of OPVs, the detailed parameters dependence on the annealing time are listed in Table 2. The annealing treatment conditions have apparent effect on the performance of OPVs.

Lin et al. [46] reported the electric field-assisted selforganization of polymer:fullerene hybrids to improve the photovoltaic performance. The "as-casted" device exhibits photovoltaic performance with a $J_{\mathrm{sc}}$ of $6.14 \mathrm{~mA} / \mathrm{cm}^{2}, V_{\mathrm{oc}}$ of $0.53 \mathrm{~V}$, and FF of $46 \%$, resulting in a PCE of $1.51 \%$ under AM $1.5\left(100 \mathrm{~mW} / \mathrm{cm}^{2}\right)$ illumination. The device prepared under the solvent-only annealing exhibits an improved performance with $J_{\mathrm{sc}}$ of $8.23 \mathrm{~mA} / \mathrm{cm}^{2}, V_{\mathrm{oc}}$ of $0.57 \mathrm{~V}$, and FF of $51 \%$, resulting in a PCE of $2.37 \%$. As the applied electric field is increased up to $6 \times 10^{3} \mathrm{~V} \mathrm{~cm}^{-1}$, a systematic enhancement in photovoltaic performance can be observed, giving the best PCE of $4.1 \%$ with the values of $J_{\mathrm{sc}}, V_{\mathrm{oc}}$ and FF respectively $11.60 \mathrm{~mA} / \mathrm{cm}^{2}, 0.59 \mathrm{~V}$ and $60 \%$. A further increase in the electric field results in a decline of the photovoltaic performance of the devices. The electric field-assisted

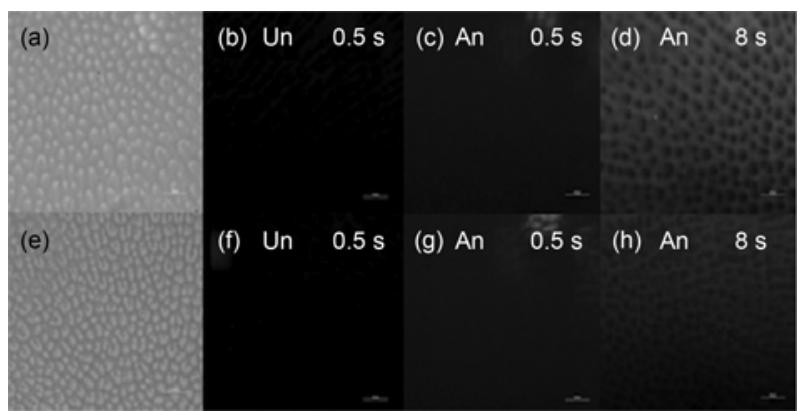

Figure 3 The optical microscopy of P3HT:PC ${ }_{60} \mathrm{BM}$ (a)-(d) and P3HT: $\mathrm{PC}_{70} \mathrm{BM}$ (e)-(f). Images (a) and (e) are under white light; other images are under green light excitation. Un, Before annealing treatment; an, after annealing treatment; 0.5 and $8 \mathrm{~s}$ are the exposure time. Reprinted with permission from [28]. Copyright (C) 2012, Elsevier.

Table 2 Photovoltaic characterization data for PSBTBT:PCBM-based OPV cells ${ }^{\text {a) }}$

\begin{tabular}{lcccc}
\hline \multicolumn{1}{c}{ Treatment } & PCE $(\%)$ & $V_{\text {oc }}(\mathrm{V})$ & $J_{\text {sc }}\left(\mathrm{mA} \mathrm{cm}^{-2}\right)$ & FF $(\%)$ \\
\hline As spun & $4.27 \pm 0.16$ & $0.59 \pm 0.01$ & $14.9 \pm 0.4$ & $48.8 \pm 1.3$ \\
$150^{\circ} \mathrm{C}, 5 \mathrm{~s}$ & $4.91 \pm 0.14$ & $0.61 \pm 0.01$ & $15.3 \pm 0.5$ & $52.8 \pm 1.3$ \\
$150^{\circ} \mathrm{C}, 30 \mathrm{~s}$ & $5.03 \pm 0.05$ & $0.59 \pm 0.01$ & $15.8 \pm 0.2$ & $53.6 \pm 1.1$ \\
$150^{\circ} \mathrm{C}, 1 \mathrm{~min}$ & $5.17 \pm 0.09$ & $0.60 \pm 0.01$ & $16.4 \pm 0.3$ & $52.8 \pm 0.9$ \\
$150^{\circ} \mathrm{C}, 5 \mathrm{~min}$ & $4.61 \pm 0.13$ & $0.59 \pm 0.01$ & $15.7 \pm 0.4$ & $49.4 \pm 1.3$ \\
$150^{\circ} \mathrm{C}, 10 \mathrm{~min}$ & $4.19 \pm 0.41$ & $0.58 \pm 0.01$ & $15.1 \pm 0.9$ & $47.3 \pm 2.7$ \\
$150^{\circ} \mathrm{C}, 20 \mathrm{~min}$ & $3.73 \pm 0.30$ & $0.60 \pm 0$ & $14.1 \pm 0.4$ & $44.2 \pm 2.9$ \\
\hline
\end{tabular}

a) Reprinted with permission from [51]. Copyright (C) 2012, Wiley VCH. 
annealing method provides a new route to fabricate polymer solar cells at low temperatures. Ma et al. [52] reported the active layer morphology was modified by an external electric field during the solution process, which provided guidance for morphology engineering in organic materials for higher performance OPVs. The direction of the applied electric field could control the vertical phase separation of P3HT: PCBM layers. The treatment of the backward electric field also reduced the tendency for self-assembled PCBM clusters to form on the surface, as shown in Figure 4. Inducing the electric field causes the polarity of the organic polymer materials to align in an ordered fashion so that the PCBM molecules will disperse and will not form cluster structures. It is interesting that the devices with horizontal electric field show the best performance compared to the devices with vertical electric field and the devices without electric field treatment [53]. Therefore, more systemic researches should be carried out to elucidate the effect of applied electric field on the performance of OPVs.

\subsection{Interfacial engineering}

For organic photovoltaic cells, the interfacial problem is a crucial issue on the interfacial band offset, carrier charge transport, collection and molecular arrangement on different films. Ideal buffer layers for OPVs should possess the following properties and satisfy multiple criteria: (i) not absorb incident solar illumination, (ii) have proper energy levels so as to inject or collect only a single charge carrier type, (iii) control the molecular arrangement to increase the photon harvesting, and (iv) increase the work function of ITO substrates. Cheng et al. [54] used CuI to control the molecular orientation of copper phthalocyanine $(\mathrm{CuPc})$ and modify the anodes in organic solar cells based on $\mathrm{CuPc} / \mathrm{C}_{60}$. By introducing a $\mathrm{CuI}$ buffer between indium tinoxide and $\mathrm{CuPc}$, the PCE is significantly enhanced by a factor of $70 \%$. Because of strong interactions between the $\mathrm{CuI}$ and $\mathrm{CuPc}$, the stacking orientation of $\mathrm{CuPc}$ molecules is changed, resulting in a $65 \%$ increase in absorption coefficient, larger carrier mobility and a smoother film surface. The anode work function is raised by the formation of a dipole layer. Yook et al. [55] reported that hexaazatriphenylene-hexacarbonitrile (HAT-CN) can be used as a templating material that forces the CuPc donor molecule to assume a vertical-standing morphology when deposited onto its surface on ITO substrates. For a device with HAT-CN as the templating buffer layer, the FF and $J_{\mathrm{sc}}$ of $\mathrm{CuPc}: \mathrm{C}_{60}$ OPVs were both significantly increased compared with cells lacking the HAT-CN template. This is explained by the reduction of the series resistance due to the improved crystallinity of $\mathrm{CuPc}$ grown on the ITO surface.

He et al. [21] have successfully demonstrated the simultaneous enhancement in the $V_{\text {oc }}, J_{\text {sc }}$ and FF by simply incorporating a thin layer of alcohol/water-soluble polymer as the cathode interlayer, resulting in a PCE up to $6.79 \%$ and a certified $8.37 \%$ for PCDTBT and PTB7 devices, respectively. The effects of the interlayer on the improvement of device performance are shown to be threefold: (i) an enhanced built-in potential across the device due to the existence of interface dipole, (ii) improved charge-transport properties, elimination of the buildup of space charge, and (iii) reduced recombination loss due to the increase in built-in field and charge carrier mobility.

Brett's group [56-58] reported a series of landmark research works to fabricate nanostructured films by glancing angle deposition (GLAD). Using high surface area nanostructured films in OPV cells is an efficient route to enhanced PCE. The diagram of GLAD equipments and prepared nanostructured films are shown in Scheme 1 [56]. The GLAD is an established single step physical vapor-deposition technique to fabricate nanostructured thin films from a full range of organic semiconductor, and dielectric materials. The two key parameters of GLAD that describe the film growth conditions are the angle between incident vapor flux and the substrate normal and the substrate rotation, which influences the characteristic columnar structures that impart OPV cells with unique and useful properties.

Constructing the optimal BHJ at the nanoscale is challenging and various fabrication methods have been applied in pursuing this architecture. Many attempts rely on the natural segregation of polymer blends while others use co-deposition of materials, both of which permit the formation of isolated regions of material and charge trapping zones. Nevertheless the achievements made using these techniques are a significant improvement over the original
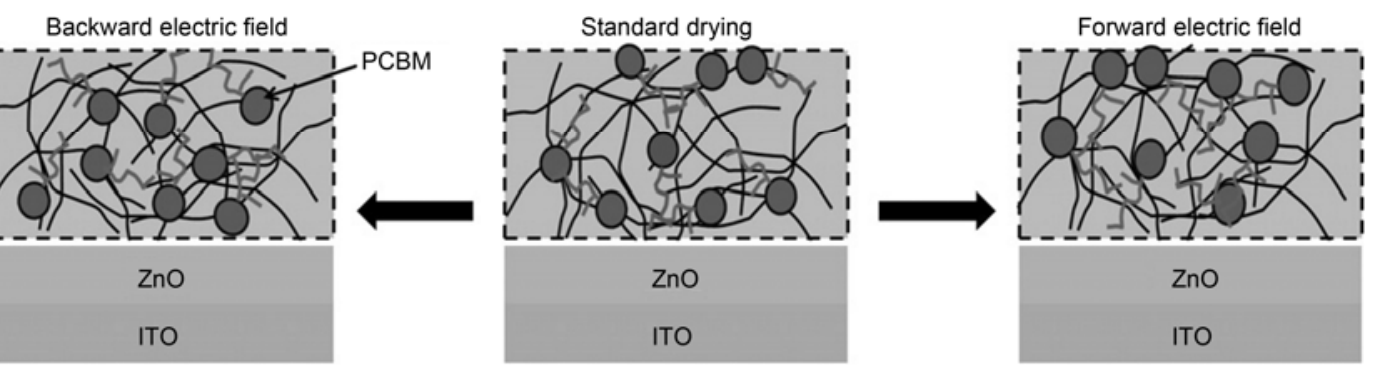

Figure 4 The schematic representation of PCBM distributions treating with different direction electric fields. When treating with a backward electric field, the PCBM side chains are aligned upward and restrain the diffusion of PCBM molecules to the top surface. When treating with a forward electric field, it will have the opposite effect to the PCBM and still forms a cluster structure on the surface. Reprinted with permission from [52]. Copyright $\odot$ 2012, Elsevier. 
(a) GLAD of ITO columns



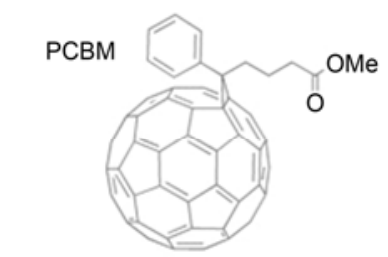

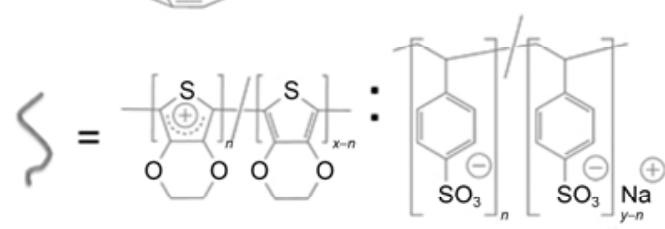

PEDOT:PSS
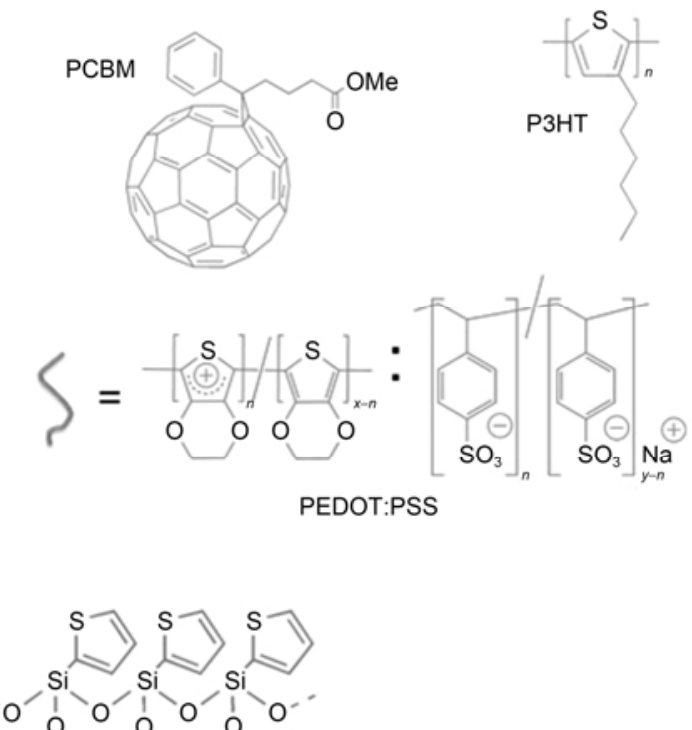
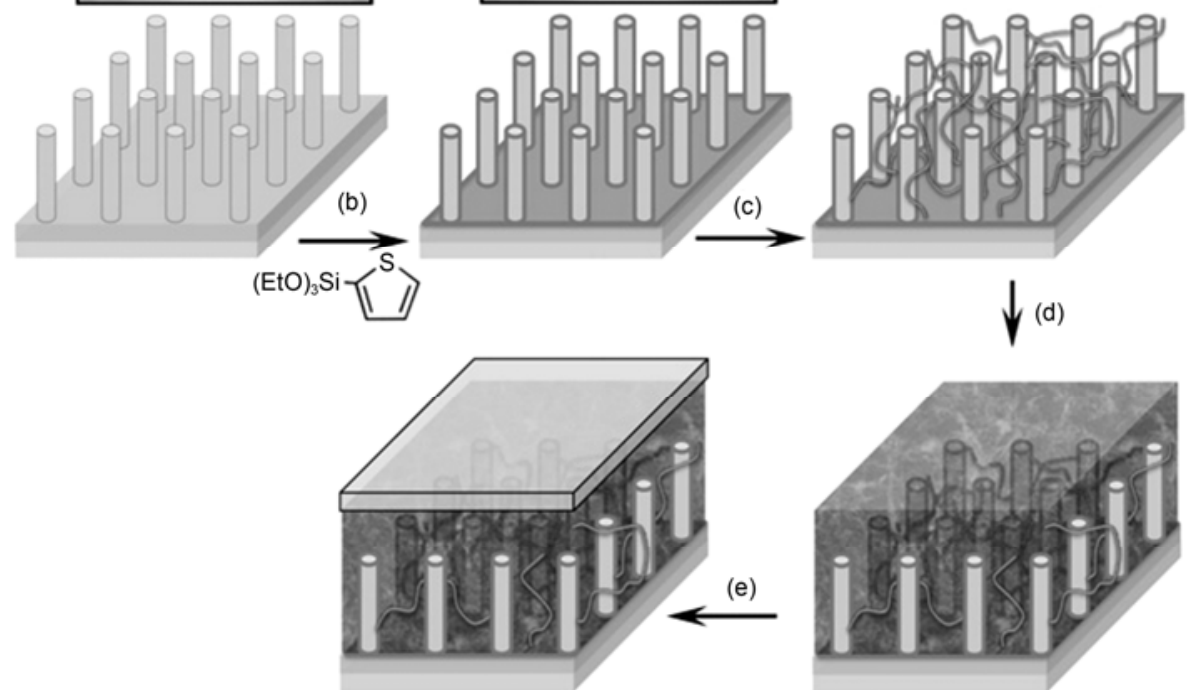
$\downarrow$ (d)

e)

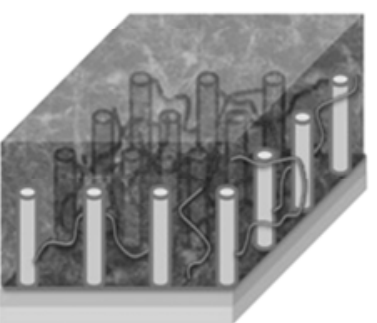

Scheme 1 Schematic illustrating the fabrication of polymer/fullerene solar cells from high surface area indium tin oxide nanoelectrodes coated with cobweb-like PEDOT:PSS. (a) Glancing angle deposition of ITO columns; (b) monolayer functionalization; (c) electrodeposition of PEDOT:PSS; (d) spin-coating 1:1 P3HT/PCBM photoactive layer; (e) evaporation of aluminum top contact. Reprinted with permission from [56]. Copyright @2012, IOP Publishing Ltd.

bilayer cell. Applying these nanostructured $\mathrm{C}_{60}$ prepared by glancing angle deposition into P3CBT as donor materials, the engineered nanomorphology yielded a 5-fold increase in $J_{\text {sc }}$ and a 4-fold increase in PCE compared with a planar cells with the same materials [57].

\subsection{Solvent and additive choice}

Adopting solvent additive is one simple and efficient way to control the blend morphology and phase separation for high PCE, which involves the addition of a small fraction of high boiling point solvent into the blend of donor/acceptor dissolved in another host solvent. A few criteria have since been empirically proposed to simplify the selection of the appropriate solvent additives. First, the solvent additive should have a higher boiling point than that of the host solvent as it allows the additive molecules to stay longer in the blend to interact with the active BHJ components upon the evaporation of the host solvent during spin-coating. Second, the additive should have a preferential solubility with either one of the D/A molecules. The effective additives for the improvement of OPVs are listed in Table 3 [21,24,62-69]. The different extents of the molecule aggregation can be correlated to the degree of intermolecular interactions between the additive molecules and the active materials, which depend on the solubility parameter and the boiling point of the solvents. The solubility parameter, which is often used to estimate the miscibility between organic solvents, is used to estimate the interaction strength. However, the duration of interaction is directly related to the boiling point 
Table 3 The effective additives for the improvement of OPVs

\begin{tabular}{|c|c|c|c|c|c|}
\hline \multirow{2}{*}{ No. } & \multirow{2}{*}{ Additive } & \multirow{2}{*}{ Active layer } & \multicolumn{2}{|c|}{ PCE (\%) } & \multirow{2}{*}{ Ref. } \\
\hline & & & Without & With & \\
\hline 1 & ATMB & P3HT:PC ${ }_{60} \mathrm{BM}$ & 1.29 & 5.03 & {$[62]$} \\
\hline 2 & $\mathrm{LC}$ & P3HT:PC 60 BM & 1.1 & 2.9 & [63] \\
\hline 3 & DLC & P3HT:PC ${ }_{60} \mathrm{BM}$ & 3.03 & 3.97 & [64] \\
\hline 4 & DIO & HXS-1:PC ${ }_{71} \mathrm{BM}$ & 3.6 & 5.4 & {$[65]$} \\
\hline 5 & DIO & PTTBDT-C8:PC ${ }_{71} B M$ & 1.45 & 4.66 & [66] \\
\hline 6 & DIO & PTB7:PC ${ }_{71} \mathrm{BM}$ & 6.22 & 7.18 & [21] \\
\hline 7 & $\mathrm{I}_{2}$ & P3HT:PCBM & 1.09 & 1.51 & [41] \\
\hline 8 & DPA & P3HT: $\mathrm{PC}_{60} \mathrm{BM}$ & 0.66 & 1.09 & [67] \\
\hline 9 & Alkyl thiol & P3HT:PC ${ }_{60} \mathrm{BM}$ & 0.9 & 2.4 & [68] \\
\hline 10 & Nitrobenzene & PDTSTPD:PC ${ }_{71} \mathrm{BM}$ & - & 6.7 & [69] \\
\hline
\end{tabular}

of the additive. Yao et al. [59] demonstrated that the high boiling point of the solvent additive is an important factor in their proposed post solvent removal mechanism, whereby the poor interaction between additive molecules and the active material components resulted in enhanced phase separation and hence the formation of an interpenetrating network in the blend film.

The use of processing additives in the optimization of the photovoltaic performance has been demonstrated to be an effective method to improve the PCE of OPVs $[3,60,61]$. In the case of $\mathrm{P} 3 \mathrm{HT} / \mathrm{PCBM}$ blends, the processing additive appears to enhance the photo-responsivity in the blend films, increase the charge carrier mobility and increase the crystallinity of the P3HT phase. In the case of low bandgap polymer/PCBM blends, the enhancement in photovoltaic properties is attributed to the increased charge carrier generation efficiency as a result of the formation of a bi-continuous nano-morphology.

\section{Stability and lifetime of OPVs}

Apart from the efficiency of OPVs, the stability and lifetime of cells are also the key problems for the realization of mass production and application of OPVs. The stability of materials determines the lifetime and stability of OPVs under oxygen, humidity, temperature as well as light conditions. In 2011, the lifetime of polymer-based solar cell with encapsulation has arrived to about 7 years [16]. For an intrinsic lifetime of organic photovoltaic devices, materials and electrodes need to be stable even without any encapsulation. An alternative approach to make devices intrinsically more stable is to adopt an inverted configuration where ITO serves as the cathode and a high work function metal as the anode [70]. Performances of inverted devices are quite stable even without any encapsulation. Hau et al. [71] reported that the inverted device structure possessed much better stability under ambient conditions retaining over $80 \%$ of its original conversion efficiency after $40 \mathrm{~d}$, while the conventional one showed a negligible photovoltaic activity after
$4 \mathrm{~d}$. Figure 5 shows the $J-V$ characteristics of conventional and inverted configuration OPVs on the storage time. Lai et al. [72] synthesized a new $\mathrm{C}_{60}$ derivative, [6,6]-phenyl$\mathrm{C}_{61}$-butyric acid 2-(2',2":5", $2^{\prime \prime \prime}$-terthiophene-5'-yl)ethyl ester (PCBTTE), and blended it into the BHJ film. PCBTTE acting as a surfactant can reduce the contact resistance after undergoing chemical reactions with the $\mathrm{Al}$ atoms of the cathode and enhance the stability of polymer solar cells. That organic materials and metals used as the electrodes react with oxygen and water which are diffused from both electrodes and lateral of the device is believed as the major reason causing short lifetime of OPVs. The underlying reason for the degradation of OPVs should be investigated. Using time-of-flight secondary ion mass spectrometry, Krebs et al. [73] demonstrated that the oxygen was diffused into the $\mathrm{Al}$ electrode through $\mathrm{Al}$ grains and microscopic holes on the Al film. Once inside the devices, oxygen was
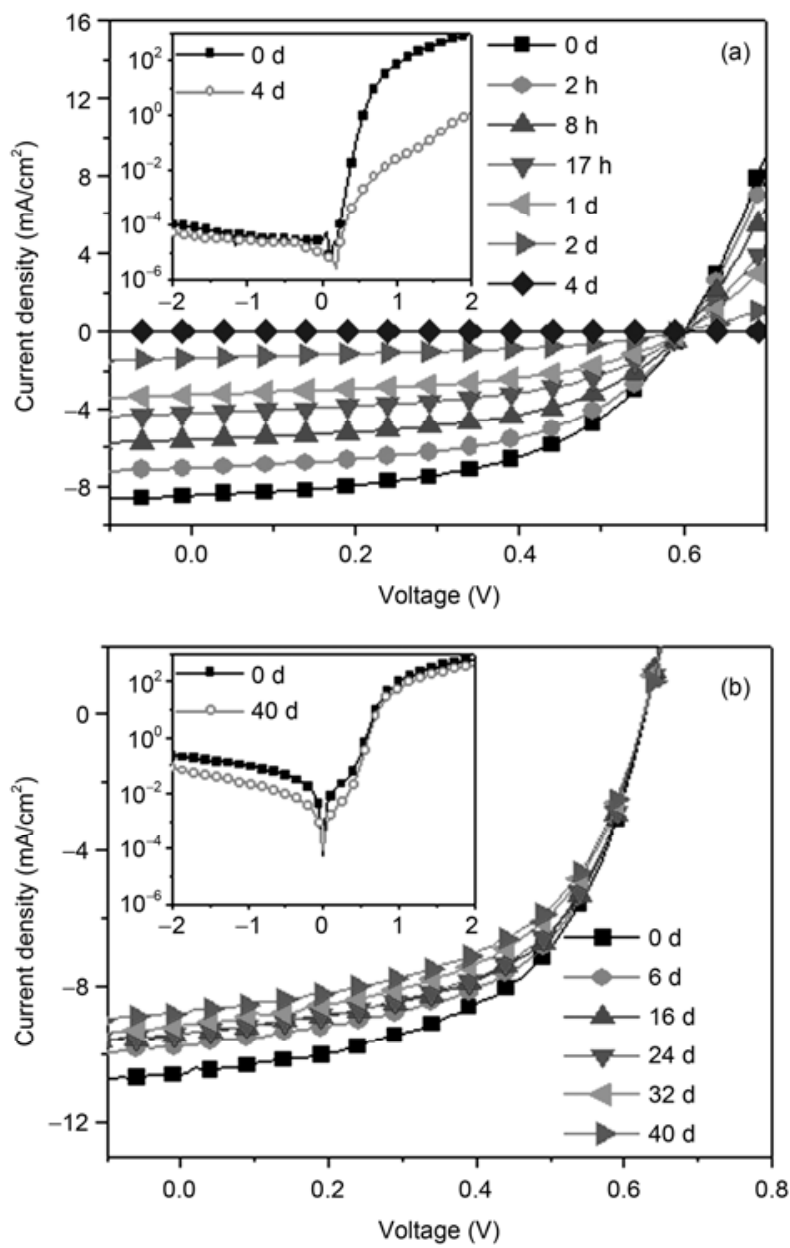

Figure 5 (a) $J-V$ characteristics of unencapsulated conventional P3HT: PCBM bulk-heterojunction solar cells over a period of $4 \mathrm{~d}$ in air under ambient conditions. (b) $J-V$ characteristics of unencapsulated inverted P3HT: PCBM bulk-heterojunction solar cells ( $\mathrm{ZnO}$ NPs on ITO-coated plastic substrate) over a period of $40 \mathrm{~d}$ in air under ambient conditions. Inset: unencapsulated dark current device characteristics at 0 and $40 \mathrm{~d}$ in air under ambient conditions. Reprinted with permission from [71]. Copyright (C) 2012, American Institute of Physics. 
continually diffused in the lateral and vertical plane until reaching the counter electrode. Another prominent degradation pathway was found to be the diffusion of electrode materials into the devices. The main degradation is due to the interfacial variation inside the cells. So the interfacial engineering is a key issue for the improvement of performance and stability of organic electronic devices.

\section{Conclusions and outlook}

Molecular engineering usually aims to achieve polymer with tunable HOMO and LUMO levels, which could improve the photon harvest and decrease the potential loss. Another important issue is to control the molecular alignment resulting in the better light absorption and charge carrier transport. Problems we should face in optimizing materials at single molecular level, small changes in a single molecule may result in large effect on the molecular electronic performance. Some novel characterization technologies should be developed for further understanding on single molecular property. OPVs have experienced a steady increase in PCE exceeding 9\%. It is reasonable to argue that OPVs within the limited years will be able to challenge the polycrystalline silicon cells if this increase trend will be kept. It was shown by the company Konarka that encapsulated large area photovoltaic devices were able to show a high performance on a roof-top for almost 2 years. Mitsubishi Chemical has developed printable solar cells with a PCE of $9.2 \%$. The OPVs will be a prime candidate for future low cost solar energy generation.

This work was supported by the National Natural Science Foundation of China (10804006, 20904057 and 21074055), the Natural Science Foundation of Beijing (2122050), the Basic Research Foundation of the Central Universities (2011JBM123). F. Zhang thanks the support from the "Double Hundred Talents Plan" of Beijing Jiaotong University and the State Key Laboratory of Catalysis, Dalian Institute of Chemical Physics, Chinese Academy of Sciences.

1 Mariano C Q, Toby F, Tiziano A, et al. Morphology evolution via self-organization and lateral and vertical diffusion in polymer: Fullerene solar cell blends. Nat Mater, 2008, 7: 158-164

2 Tang C W. Two layer organic photovoltaic cell. Appl Phys Lett, 1986, 43: $183-185$

3 Peet J, Kim J Y, Coates N E, et al. Efficiency enhancement in lowbandgap polymer solar cells by processing with alkane dithiols. Nat Mater, 2007, 6: 497-500

4 Jin Y K, Kwanghee L, Nelson E C, et al. Efficient tandem polymer solar cells fabricated by all-solution processing. Science, 2007, 13: 222-225

5 Dennler G, Scharber M C, Brabec C J, et al. Polymer fullerene bulk heterojunction solar cells. Adv Mater, 2009, 21: 1323-1338

6 Koster L J A, Mihailetchi V D, Blom P W M. Bimolecular recombination in polymer/fullerene bulk heterojunction solar cells. Appl Phys Lett, 2006, 88: 052104

7 Zhao D W, Sun X W, Jiang C Y, et al. Efficient tandem organic solar cells with an $\mathrm{Al} / \mathrm{MoO}_{3}$ intermediate layer. Appl Phys Lett, 2008, 93: 083305.

8 Sun X W, Zhao D W, Ke L, et al. Inverted tandem organic solar cells with $\mathrm{MoO}_{3} / \mathrm{Ag} / \mathrm{Al} / \mathrm{Ca}$ intermediate layer. Appl Phys Lett, 2010, 97 : 053303

9 Zimmermann B, Wurfel U, Niggemann M. Longterm stability of efficient inverted P3HT:PCBM solar cells. Sol Energy Mater Sol Cells, 2009, 93: 491-496

10 Zhang F J, Xu X W, Tang W H, et al. Recent development of the inverted configuration organic solar cells. Sol Energy Mater Sol Cells, 2011, 95: 1785-1799

11 Kim D H, Park Y D, Jiang Y, et al. Enhancement of field-effect mobility due to surface mediated molecular ordering in regioregular polythiophene thin film transistors. Adv Funct Mater, 2005, 15: 77-82

12 Hiorns R C, Bettignies R D, Leroy J, et al. High molecular weights, polydispersities, and annealing temperatures in the optimization of bulk heterojunction photovoltaic cells based on poly(3-hexylthiophene) or poly(3-butylthiophene). Adv Funct Mater, 2006, 16: 2263-2273

13 Zhao G J, He Y J, Li Y F, et al. 6.5\% Efficiency of polymer solar cells based on poly(3-hexylthiophene) and indene- $\mathrm{C}_{60}$ bisadduct by device optimization. Adv Mater, 2010, 22: 4355-4358

14 Sun Y M, Takacs C J, Cowan S R, et al. Efficient, air-stable bulk heterojunction polymer solar cells using $\mathrm{MoO}_{x}$ as the anode interfacial layer. Adv Mater, 2011, 23: 2226-2230

15 Park S H, Roy A, Beaupré S, et al. Bulk heterojunction solar cells with internal quantum efficiency approaching $100 \%$. Nat Photonics, 2009, 3: 297-302

16 Peters C H, Sachs-Quintana I T, Kastrop J P, et al. High efficiency polymer solar cells with long operating lifetimes. Adv Energy Mater, 2011, 1: 491-494

17 Zuo L J, Jiang X X, Xu M S, et al. Enhancement of short current density in polymer solar cells with phthalocyanine tin (IV) dichloride as interfacial layer. Sol Energy Mater Sol Cells, 2011, 95: 2664-2669

18 Wang D H, Park K H, Seo J H, et al. Enhanced power conversion efficiency in PCDTBT/PC70BM bulk heterojunction photovoltaic devices with embedded silver nanoparticle clusters. Adv Energy Mater, 2011, 1: 766-770

19 Beiley Z M, Hoke E T, Noriega R, et al. Morphology-dependent trap formation in high performance polymer bulk heterojunction solar cells. Adv Energy Mater, 2011, 1: 954-962

20 Liang Y Y, Xu Z, Xia J B, et al. For the bright future-Bulk heterojunction polymer solar cells with power conversion efficiency of 7.4\%. Adv Mater, 2010, 22: E135-E138

21 He Z C, Zhong C M, Huang X, et al. Simultaneous enhancement of open-circuit voltage, short-circuit current density, and fill factor in polymer solar cells. Adv Mater, 2011, 23: 4636-4643

22 Chu T Y, Lu J, Beaupré S, et al. Bulk heterojunction solar cells using thieno 3,4-cpyrrole-4,6-dione and dithieno 3,2-b:2',3'-d silole copolymer with a power conversion efficiency of $7.3 \%$. J Am Chem Soc, 2011, 133: 4250-4253

23 Chen H Y, Hou J H, Zhang S Q, et al. Polymer solar cells with enhanced open-circuit voltage and efficiency. Nat Photonics, 2009, 3: 649-653

24 Walker B, Arnold B, Tamayo, et al. Nanoscale phase separation and high photovoltaic efficiency in solution-processed, small-molecule bulk heterojunction solar cells. Adv Funct Mater, 2009, 19: 3063-3069

25 Placencia D, Wang W, Shallcross R C, et al. Organic photovoltaic cells based on solvent-annealed, textured titanyl phthalocyanine/ $\mathrm{C}_{60}$ heterojunction. Adv Funct Mater, 2009, 19: 1913-1921

26 Kageyama H, Ohishi H, Tanaka M, et al. High-performance organic photovoltaic devices using a new amorphous molecular material with high hole drift mobility, tris 4-(5-phenylthiophen-2-yl)phenyl amine. Adv Funct Mater, 2009, 19: 3948-3955

27 Liang Y Y, Wu Y, Feng D Q, et al. Development of new semiconducting polymers for high performance solar cells. J Am Chem Soc, 2009, 131: 56-57

28 Zhang F J, Zhuo Z L, Zhang J, et al. Influence of PC60BM or PC70BM as electron acceptor on the performance of polymer solar cells. Sol Energy Mater Sol Cells, 2012, 97: 71-77

29 Sariciftci N S, Smilowitz L, Heeger A J, et al. Photoinduced electron transfer from a conducting polymer to buckminsterfullerene. Science, 1992, 258: 1474-1476

30 Pandey A K, Shaw P E, Samuel D W, et al. Effect of metal cathode 
reflectance on the exciton-dissociation efficiency in heterojunction organic solar cells. Appl Phys Lett, 2009, 94: 103303

31 Schinazi R F, Sijbesma R, Srdanov G, et al. Synthesis and virucidal activity of a water-soluble, configurationally stable, derivatived C60 Fullerene. Antimicrob Agents Chemother, 1993, 37: 1707-1710

32 Zhao G J, He Y J, Xu Z, et al. Effect of carbon chain length in the substituent of PCBM-like molecules on their photovoltaic properties. Adv Funct Mater, 2010, 20: 1480-1487

33 Morvillo P. Higher fullerenes as electron acceptors for polymer solar cells: A quantum chemical study. Sol Energy Mater Sol Cells, 2009, 93: $1827-1832$

34 He Y J, Chen H Y, Hou J H, et al. Indene-C60 bisadduct: A new acceptor for high-performance polymer solar cells. J Am Chem Soc, 2010, 132: 1377-1382

35 Chen C P, Lin Y W, Horng J C, et al. Open cage fullerenes as n-type materials in organic photovoltaics: Relevance of frontier energy levels, carrier mobility and morphology of different sizable open cage fullerenes with power conversion efficiency in devices. Adv Energy Mater, 2011, 1: 776-780

36 Kooistra F B, Mihailetchi V D, Popescu L M, et al. New $\mathrm{C}_{84}$ derivative and its application in a bulk heterojunction solar cell. Chem Mater, 2006, 18: 3068-3073

37 Ross R B, Cardona C M, Guldi D M, et al. Endohedral fullerenes for organic photovoltaic devices. Nat Mater, 2009, 8: 208-212

38 Ren G Q, Ahmed E, Jenekhe S A, et al. Non fullerene acceptor based bulk heterojunction polymer solar cells: Engineering the nanomorphology via processing additives. Adv Energy Mater, 2011, 1: 946-953

39 Zhuo Z L, Zhang F J, Xu X W, et al. Photovoltaic performance improvement of P3HT:PCBM polymer solar cells by annealing treatment. Acta Phys Chim Sin, 2011, 27: 875-880

40 Zhao D W, Sun X W, Jiang C Y, et al. An efficient triplet tandem polymer solar cell. IEEE Electron Dev Lett, 2009, 30: 490

41 Zhuo Z L, Zhang F J, Wang J, et al. Efficiency improvement of polymer solar cells by iodine doping. Solid-State Electron, 2011, 63: 83-88

42 Xie F X, Choy W C H, Zhu X L, et al. Improving polymer solar cell performances by manipulating the self organization of polymer. Appl Phys Lett, 2011, 98: 243302

43 Zhang F J, Sun F Y, Shi Y Z, et al. Effect of an ultra-thin molybdenum trioxide layer and illumination intensity on the performance of organic photovoltaic devices. Energy Fuels, 2010, 24: 3739-3742

44 Xu X W, Zhu E W, Bian L Y, et al. Luminescent and photovoltaic properties of poly(9,9-dioctylfluorene-co-bithiophene) in organic electronic devices. Chin Sci Bull, 2012, 57: 970-973

45 Bagienski W, Gupta M C. Temperature dependence of polymer/fullerene organic solar cells. Sol Energy Mater Sol Cells, 2011, 95: 933-941

46 Lin C C, Lin Y Y, Li S S, et al. Electric field-assisted self-organization of polymer: Fullerene hybrids on the photovoltaic performance. Energy Environ Sci, 2011, 4: 2134-2139

$47 \mathrm{Li} \mathrm{H}$, Tang H W, Li L G, et al. Solvent-soaking treatment induced morphology evolution in P3HT/PCBM composite films. J Mater Chem, 2011, 21: 6563-6568

48 Peng R X, Zhu J, Pang W M, et al. Thermal annealing effects on the absorption and structural properties of regioregular poly(3-hexylthiophene) films. J Macromol Sci Part B-Phys, 2011, 50: 624-636

49 Chiguvare Z, Dyakonov V. Trap-limited hole mobility in semiconducting poly(3-hexylthiophene). Phys Rev B, 2004, 70: 235207

50 Ma W L, Yang C Y, Gong X, et al. Thermally stable, efficient polymer solar cell with Nanoscale control of the interpenetrating network morphology. Adv Funct Mater, 2005, 15: 1617-1622

51 Lu H Y, Akgun B, Russell T P. Morphological characterization of a low-bandgap crystalline polymer: PCBM bulk heterojunction solar cells. Adv Energy Mater, 2011, 1: 870-878

52 Ma S Y, Shen Y M, Yang P C, et al. Morphology modification induced by external electric field during solution process of organic solar cells. Org Electron, 2012, 13: 297-301
53 Shen Y M, Chen C S, Yang P C, et al. Improvement of surface morphology of thin films and performance by applying electric field on P3HT: PCBM based solar cells. Sol Energy Mater Sol Cells, 2011, 99: 263-267

54 Cheng C H, Wang J, Du G T. Organic solar cells with remarkable enhanced efficiency by using a CuI buffer to control the molecular orientation and modify the anode. Appl Phys Lett, 2010, 97: 083305

55 Yook K S, Chin B D, Lee J Y. Vertical orientation of copper phthalocyanine in organic solar cells using a small molecular weight organic templating layer. Appl Phys Lett, 2011, 99: 043308

56 Rider D A, Tucker R T, Worfolk B J, et al. Indium tin oxide nanopillar electrodes in polymer/fullerene solar cells. Nanotechnology, 2011, 22: 085706

57 Thomas M, Worfolk B J, Rider D A, et al. $\mathrm{C}_{60}$ fullerene nanocolumns-polythiophene heterojunctions for inverted organic photovoltaic cells. ACS Appl Mater Interfaces, 2011, 3: 1887-1894

58 Dijken J G V, Fleischauer M D, Brett M J, et al. Controlled nanostructuring of $\mathrm{CuPc}$ thin films via glancing angle deposition for idealized organic photovoltaic architectures. J Mater Chem, 2011, 21: 1013-1019

59 Yao Y, Hou J, Xu Z, et al. Effects of solvent mixtures on the nanoscale phase separation in polymer solar cells. Adv Funct Mater, 2008, 18: 1783-1789

60 Chen H Y, Yang H C, Yang G W, et al. Fast-grown interpenetrating network in poly(3-hexylthiophene): Methanofullerenes solar cells processed with additive. J Phys Chem C, 2009, 113: 7946-7953

61 Lee J K, Ma W L, Brabec C J, et al. Processing additives for improved efficiency from bulk heterojunction solar cells. J Am Chem Soc, 2008, 130: 3619-3623

62 Jeong S, Woo S H, Lyu H K, et al. Effects of a perfluorinated compound as an additive on the power conversion efficiencies of polymer solar cells. Sol Energy Mater Sol Cells, 2011, 95: 1908-1914

63 Yilmaz C N. Chiral (S)-5-octyloxy-2-\{4-(2-methylbuthoxy)-phenylimino\}-methyl-phenol liquid crystalline compound as additive into polymer solar cells. Sol Energy Mater Sol Cells, 2010, 94: 1089-1099

64 Jeong S, Kwon Y H, Choi B D, et al. Improved efficiency of bulk heterojunction poly(3-hexylthiophene): 6,6-phenyl-Csub 61-butyric acid methyl ester photovoltaic devices using discotic liquid crystal additives. Appl Phys Lett, 2010, 96: 183305

$65 \mathrm{Li} \mathrm{W}$, Zhou Y, Andersson V, et al. The effect of additive on performance and shelf-stability of HSX-1/PCBM photovoltaic devices. Org Electron, 2011, 12: 1544-1551

66 Zhang Y, Zhao L, Wakim S, et al. Bulk heterojunction solar cells based on a new low-band-gap polymer: Morphology and performance. Org Electron, 2011, 12: 1211-1215

67 Ismail Y A M, Soga T, Jimbo T, et al. Improvement in light harvesting and performance of P3HT:PCBM solar cell by using 9,10-diphenylanthracene. Sol Energy Mater Sol Cells, 2009, 93: 1582-1586

68 Pivrikas A, Stadler P, Neugebauer H, et al. Substituting the postproduction treatment for bulk-heterojunction solar cells using chemical additives. Org Electron, 2008, 9: 775-782

69 Chu T Y, Tsang S W, Zhou J Y, et al. High-efficiency inverted solar cells based on a low bandgap polymer with excellent air stability. Sol Energy Mater Sol Cells, 2012, 96: 155-159

$70 \mathrm{Xu} \mathrm{X} \mathrm{W,} \mathrm{Zhang} \mathrm{F} \mathrm{J,} \mathrm{Zhang} \mathrm{J,} \mathrm{et} \mathrm{al.} \mathrm{High} \mathrm{efficient} \mathrm{inverted} \mathrm{polymer}$ solar cells with different annealing treatment. Mater Sci Eng C, 2012, 32: 685-691

71 Hau S K, Yip H L, Baek N S, et al. Air-stable inverted flexible polymer solar cells using zinc oxide nanoparticles as an electron selective layer. Appl Phys Lett, 2008, 92: 253301

72 Lai Y C, Tomoya H, Hsu J C, et al. Enhancement of power conversion efficiency and long-term stability of P3HT/PCBM solar cells using $\mathrm{C}_{60}$ derivatives with thiophene units as surfactants. Sol Energy Mater Sol Cells, 2012, 97: 164-170

73 Krebs F C, Norrman K. Analysis of the failure mechanism for a stable organic photovoltaic during $10000 \mathrm{~h}$ of testing. Prog Photovolt: Res Appl, 2007, 15: 697-712

Open Access This article is distributed under the terms of the Creative Commons Attribution License which permits any use, distribution, and reproduction in any medium, provided the original author(s) and source are credited. 